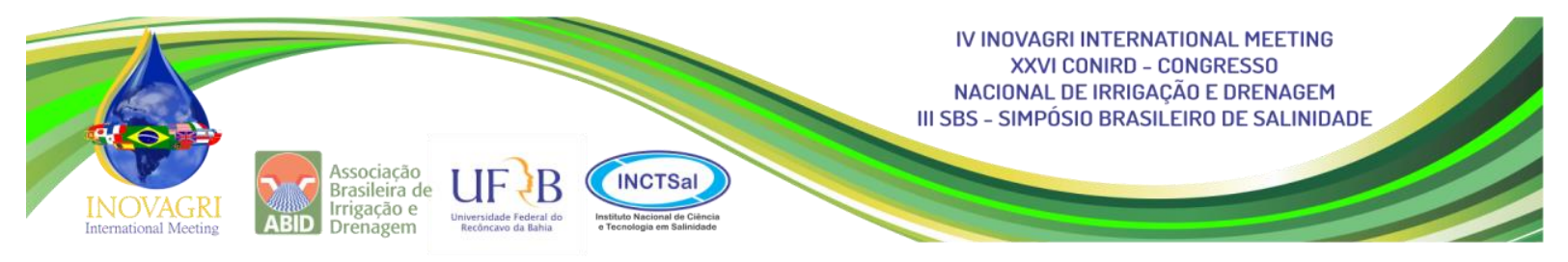

\title{
IRRIGAÇÃO DE CEBOLA POR GOTEJAMENTO NA SUPERFÍCIE DO SOLO E SUBTERRÂNEO
}

\author{
J. M. Pinto ${ }^{1}$, W. L. Simões ${ }^{2}$, M. Calgaro ${ }^{2}$
}

RESUMO: Realizou-se, no Campo Experimental de Bebedouro, pertencente à Embrapa Semiárido, em Petrolina, PE, um estudo com o objetivo de avaliar a produção de cebola, cultivar IPA 11, irrigada por gotejamento com tubos gotejadores dispostos na superfície do solo ou enterrados a 0,20 metros de profundidade. $O$ espaçamento entre gotejadores foi de 0,30 metros, vazão do emissor de $1 \mathrm{Lh}^{-1}$. O delineamento estatístico foi fatorial (2x3), blocos ao acaso, sendo duas formas de disposição de linhas laterais e número de linhas laterais por canteiro (2, 3 e 4 linhas laterais por canteiro), com cinco repetições, totalizando 30 parcelas. A dimensão da parcela foi de 1,10 metro de largura por 5,0 metros de comprimento. O espaçamento entre fileiras de plantas no canteiro foi $0,10 \mathrm{~m}$ e, entre plantas na fileira, de 0,15 m. O cálculo da lâmina de água foi realizado com base na evaporação da água do tanque classe A e coeficiente de cultivo da cultura. O cálculo das doses de N, P e K foi baseado na análise de solo. Aplicaram-se: $156 \mathrm{~kg} \mathrm{ha}^{-1}$ de ureia, $150 \mathrm{~kg} \mathrm{ha}^{-1} \mathrm{de}$ MAP, $80 \mathrm{~kg} \mathrm{ha}^{-1}$ de cloreto de potássio. O nitrogênio foi aplicado junto com o potássio, três vezes por semana, via água de irrigação, utilizando-se um injetor de fertilizante tipo venturi. A fertirrigação iniciou três dias após o transplantio e se estendeu por 70 dias. O fósforo foi aplicado em fundação, antes do plantio. O consumo de água foi de $3.760 \mathrm{~m}^{3} \mathrm{ha}^{-1}$. O rendimento médio obtido foi de 27,31; 35,88 e 32,67 t ha ${ }^{-1}$ com tubos gotejadores na superfície do solo, com 2, 3 e 4 linhas laterais por canteiro, respectivamente. Para linhas laterais subterrâneas, o rendimento foi 20,17; 28,38 e 31,42 $\mathrm{tha}^{-1}$ com 2, 3 e 4 linhas laterais por canteiro, respectivamente. Recomenda-se, para a cultura da cebola, o sistema de irrigação por gotejamento com três linhas laterais por canteiro, dispostas na superfície do solo. O sistema de irrigação por gotejamento subterrâneo com linhas laterais mostrou-se contra indicado para a cultura da cebola.

PALAVRAS-CHAVE: Allium cepa, fertirrigação, consumo de água.

\footnotetext{
${ }^{1}$ Eng. Agric., D. Sc., Pesquisador, Embrapa Semiárido, BR 428, km 152 Zona Rural, Caixa Postal 23, 56302-970 Petrolina, PE. E-mail: josemaria.pinto@embrapa.br

${ }^{2}$ Eng. Agr., D. Sc., Pesquisador, Embrapa Semiárido, BR 428, km 152 Zona Rural, Caixa Postal 23, 56302-970 Petrolina, PE
} 


\section{DRIP IRRIGATION ON SURFACE OR UNDERGROUND ON ONION CROP}

SUMMARY: The trail was conducted at the Embrapa Semiarido, in Petrolina, PE, Brazil, in a field of Bebedouro Experiment Station, to evaluate the onion production, cultivar IPA 11, irrigated by drip irrigation with dripping tubes arranged on the soil surface and buried at 0.20 meters deep. The drip spacing was 0.30 meters and the flow rate was $1 \mathrm{~L} \mathrm{~h}^{-1}$. The statistical design was a factorial randomized block design (2x3), two forms of lateral line layout and number of lateral lines per plot (2, 3 and 4 lateral lines per plot), with five replications. The total plots number was 30. The dimension of the plot was 1.10 meters wide by 5.0 meters long. The spacing between rows of plants on the plot was $0.10 \mathrm{~m}$ and between plants in the row $0.15 \mathrm{~m}$. The water amount was calculated based on the evaporation of the class A tank and culture coefficient of the culture. The N, P and K levels were based on soil analysis results. It applied $156 \mathrm{~kg} \mathrm{ha}^{-1}$ of urea, $150 \mathrm{~kg} \mathrm{ha}^{-1}$ of MAP, $80 \mathrm{~kg} \mathrm{ha}^{-1}$ of potassium chloride. Nitrogen was applied together with potassium, three times a week, through irrigation water. It used a fertilizer injector type venturi. Fertigation started three days after transplanting and extended for 70 days. The phosphorus was applied in foundation, before planting. The water consumption was $3,760 \mathrm{~m}^{3} \mathrm{ha}^{-1}$. The average yield obtained were $27.31 ; 35.88$ and 32.67 tons.ha ${ }^{-1}$ with pipe drippers at the soil surface, with 2,3 and 4 lateral lines per plot, respectively. For subterranean sidelines, yield were $20.17 ; 28.38$ and 31.42 tons.ha $^{-1}$ with 2,3 and 4 lateral lines per plot, respectively. For onion cultivation should be recommended the drip irrigation system with three lateral lines, disposed on the surface of the soil, per plot. Drip irrigation system with underground lateral lines proved contraindicated for onion culture.

KEY WORDS: Allium cepa, fertigation, water consumption.

\section{INTRODUÇÃO}

A irrigação no Brasil é realizada de forma inadequada, com desperdício de água (MANTOVANI et al., 2006). Estima-se que, de toda a água captada para fins de irrigação, apenas $50 \%$ é efetivamente utilizada pelas plantas (CHRISTOFIDIS, 2004). Em sistemas de irrigação por superfície, estima-se que não mais de 35\% da água que é retirada de fontes superficiais ou subterrâneas chega efetivamente às plantas. Segundo Mantovani et al. (2006), tal problema ocorre devido a três fatores principais: diminuta utilização de critérios técnicos de manejo na maioria das áreas irrigadas; informações escassas e incompletas sobre manejo 
de água e uso de sistemas de irrigação com baixa eficiência de aplicação de água. $\mathrm{O}$ desperdício de água verificado, além de aumentar os custos de produção, com energia, acarreta custos ambientais pelo comprometimento da disponibilidade deste recurso natural. Levado vários projetos de irrigação, em todo o mundo, a uma condição de baixa sustentabilidade econômica e socioambiental.

A irrigação por gotejamento pode ser instalada com linhas laterais na superfície do solo ou com linhas laterais subterrâneas. O gotejamento com linhas laterais subterrâneas possibilita aplicar menor lâmina de água devido à menor taxa evaporativa, maior volume de solo molhado, devido à ascensão capilar da água, redução dos danos mecânicos ocasionados pelos tratos culturais, por não necessitar a remoção do sistema de irrigação (Rajput \& Patel, 2009).

Entretanto, há a possibilidade de intrusão de raízes nos emissores, obstruindo total ou parcialmente o emissor, acarretando redução de vazão. Para evitar o problema, deve-se optar por emissores com dispositivos de proteção ou emissores antidrenantes (Souza et al., 2012).

Verifica-se aumento da fertirrigação no Brasil e no mundo, pois a técnica se mostrou efetiva no aumento de produtividade e consequentemente, no lucro obtido pelos produtores. A fertirrigação tem sido recomendada por promover o aumento da eficiência de aproveitamento de nutrientes, com diminuição da contaminação dos mananciais (PAPADOLOULOS, 1993).

É comum, em países onde a fertirrigação já vem sendo utilizada há décadas, a automação de todo o processo de fertirrigação. No Brasil, algumas empresas começam a utilizar a automação como estações de fertirrigação que permitem monitoramento e controle da condutividade elétrica e $\mathrm{pH}$, o uso de softwares de manejo de irrigação e fertirrigação (MANTOVANI et al., 2006; MAROUELLI et al., 2005; PASCHOLD et al., 2004).

A cebola (Allium cepa L.) ocupa, entre as hortaliças cultivadas, a terceira posição em importância econômica no Brasil, ficando atrás apenas da batata e do tomate (GONÇALVES et al., 2009). Em 2011, a produtividade média nacional, de acordo com o IBGE (2015), se manteve em torno de $23.278 \mathrm{~kg} \mathrm{ha}^{-1}$. Contudo, cultivos conduzidos sob irrigação podem alcançar produtividade acima de $90 \mathrm{t} \mathrm{ha}^{-1}$ (MAROUELLI et al., 2011). Shock \& Shock (2012) concluíram, em estudos realizados em Oregon, que o manejo racional de água e de nutrientes proporciona melhoria na qualidade e conservação da cebola. Sharma (1992) informou um incremento na produtividade com a aplicação de $40 \mathrm{~kg} \mathrm{ha}^{-1}$ de potássio, não se verificando nenhum efeito adicional quando se aplicaram doses superiores (80 e $160 \mathrm{~kg} \mathrm{ha}^{-1}$ de $\mathrm{K}_{2} \mathrm{O}$, respectivamente). Aumentos no diâmetro e na massa de matéria fresca do bulbo foram relatados por Mohanty \& Das (2001) com a aplicação de $60 \mathrm{~kg} \mathrm{ha}^{-1}$ de $\mathrm{K}_{2} \mathrm{O}$, enquanto Akhtar 
et al. (2002) observaram aumento na produtividade até a dose de $200 \mathrm{~kg} \mathrm{ha}^{-1}$ de $\mathrm{K}_{2} \mathrm{O}$, quando em presença de adubações nitrogenada e fosfatada.

Este trabalho teve por objetivo avaliar a influência dos métodos de irrigação por gotejamento na superfície do solo e subterrâneo e número linhas laterais por canteiro na produção de cebola IPA 11.

\section{MATERIAL E MÉTODOS}

O trabalho foi realizado com a cultura da cebola (Allium cepa L.) no Campo Experimental de Bebedouro, Petrolina, $\mathrm{PE}$, pertencente à Embrapa Semiárido. Do local do experimento, coletou-se amostra de solo na camada de $0-0,20 \mathrm{~m}$, que apresentou as seguintes características químicas: $\mathrm{pH}: 8,1$; matéria orgânica: $22,76 \mathrm{~g} \mathrm{~kg}^{-1} ; \mathrm{P}: 294 \mathrm{mg} \mathrm{dm}^{-3}, \mathrm{~K}$, $\mathrm{Ca}, \mathrm{Mg}, \mathrm{H}+\mathrm{Al}, \mathrm{Sb}: 1,85,6,9,3,2,0,12,13$, cmolc L $\mathrm{L}^{-1}$, respectivamente e V: $100 \%$.

Compararam-se dois métodos de irrigação (gotejamento na superfície do solo e subterrâneo) e número de linhas laterais (duas, três e quatro) por canteiro na cultura da cebola, cultivar IPA 11. A profundidade das linhas subterrâneas foi 0,20 metros abaixo da superfície do solo, usando mangueira de gotejador, com emissor espaçado de $0,3 \mathrm{~m}$ entre si e vazão de 1 $\mathrm{L} \mathrm{h}^{-1}$.

O delineamento estatístico foi em blocos ao acaso com cinco repetições. Cada parcela foi constituída por um canteiro de 1,10 m de largura e 5,0 $\mathrm{m}$ de comprimento. $\mathrm{O}$ espaçamento entre fileiras de plantas no canteiro foi $0,10 \mathrm{~m}$ e, entre plantas na fileira, de $0,15 \mathrm{~m}$.

A semeadura foi realizada em sementeira com largura de canteiro $(1,10 \mathrm{~m})$ e 20 metros de comprimento e 45 dias após a germinação foi feito o transplantio para os canteiros definitivos.

O cálculo da lâmina de água aplicada por irrigação foi realizado com base na evaporação da água e coeficiente de cultivo da cultura $(\mathrm{Kc})$. Foi aplicada a mesma lâmina de água em todos os tratamentos.

O cálculo das doses de $\mathrm{N}, \mathrm{P}$ e K para adubação da cebola irrigada por gotejamento foi baseado na análise de solo. Aplicaram-se $156 \mathrm{~kg} \mathrm{ha}^{-1}$ de ureia, $150 \mathrm{~kg} \mathrm{ha}^{-1}$ de MAP, $80 \mathrm{~kg} \mathrm{ha}^{-1}$ de cloreto de potássio.

O nitrogênio foi aplicado junto com o potássio, três vezes por semana, via água de irrigação, utilizando-se um injetor tipo venturi de fertilizantes. A fertirrigação iniciou aos três dias após o transplantio e se estendeu por 70 dias. O fósforo foi divido com aplicação de $30 \%$ na fundação e de $70 \%$ via água de irrigação, uma vez por semana. 
Avaliou-se a produção comercial da cebola e classificação por tamanho de bulbo (classe 1, diâmetro variando de 15 a $35 \mathrm{~mm}$; classe dois, diâmetro entre 35 e $50 \mathrm{~mm}$; classe 3 , diâmetro de 50 a 70mm; classe 4, diâmetro de 70 a 90 mm e classe 5 com diâmetro maior que 90mm) descrito por Costa et al. (2012).

\section{RESULTADOS E DISCUSSÃO}

O consumo de água irrigada por gotejamento foi de $3.760 \mathrm{~m}^{3}$ por hectare. Os dados de produtividade por tamanho de bulbo (classe 1, classe 2 e classe 3), sendo aqueles bulbos do classe 3 os de maior valor comercial (Costa et al., 2015) e produtividade comercial de cebola são apresentados nas Tabelas 1, 2, 3, para comparação dos tratamentos com as linhas laterais dispostas na superfície do solo e gotejamento com as linhas laterais subterrâneas. Na Tabela 4 são apresentados os dados de todos os tratamentos.

Apresentam-se, na Tabela 1, as produtividades de cebola por classe e a produtividade comercial para os tratamentos com irrigação por gotejamento com duas linhas laterais, com disposição na superfície do solo e subterrânea. Observou-se que o tratamento com duas linhas laterais dispostas na superfície do solo proporcionou produtividades de cebolas da classe 3 e comercial significativamente superiores às produtividades da mesma classe de bulbos obtidas no tratamento com duas linhas laterais subterrâneas. Os resultados obtidos estão de acordo com os valores encontrados por Duarte et al. (2003), que conduziram estudo com cebola irrigada Vale Ouro e IPA 1 no semiárido piauiense.

Tabela 1. Produtividade de classes 1, 2 e 3 e produtividade comercial, irrigada por gotejamento com duas linhas laterais por canteiro, dispostas na superfície do solo e enterradas a $0,20 \mathrm{~m}$.

\begin{tabular}{lcccc}
\hline \multirow{2}{*}{ Tratamento } & \multicolumn{3}{c}{ Produtividade $\left(\mathrm{t} \mathrm{ha}^{-1}\right)^{*}$} \\
\cline { 2 - 5 } & Classe 1 & Classe 2 & Classe 3 & Comercial \\
\hline Gotejamento-Superfície do solo & $5,89 \mathrm{a}$ & $12,14 \mathrm{a}$ & $9,28 \mathrm{a}$ & $27,31 \mathrm{a}$ \\
\hline Gotejamento-Subterrâneo & $5,53 \mathrm{a}$ & $12,67 \mathrm{a}$ & $1,57 \mathrm{~b}$ & $20,17 \mathrm{~b}$ \\
\hline
\end{tabular}

"Médias seguidas da mesma letra, na coluna, não diferem entre pelo teste de Scott-Knott a 5\% de probabilidade.

As produtividades de cebola por classe e a produtividade comercial para os tratamentos com irrigação por gotejamento com três linhas laterais com disposição na superfície do solo ou subterrânea são apresentadas na Tabela 2. Observou-se, pelos dados, que as maiores produtividades de bulbos classe 3 e produtividade comercial, com diferenças estatísticas significativas, foram obtidas no tratamento com três linhas laterais dispostas na superfície do 
solo. A produtividade de bulbos classe 1, de menor valor comercial, foi maior no tratamento com linhas laterais subterrâneas. Bandeira et al. (2013), avaliando o uso da irrigação por gotejamento em cebola Alfa São Francisco, IPA 10 e IPA 11 obtiveram produtividade da ordem de 40,0 $\mathrm{t} \mathrm{ha}^{-1}$ tensão de água solo igual a $30 \mathrm{kPa}$.

Tabela 2. Produtividade de classes 1, 2 e 3 e produtividade comercial, irrigada por gotejamento com três linhas laterais por canteiro, dispostas na superfície do solo e enterradas a $0,20 \mathrm{~m}$.

\begin{tabular}{lcccc}
\hline \multirow{2}{*}{ Tratamento } & \multicolumn{3}{c}{ Produtividade $\left(\mathrm{t} \mathrm{ha}^{-1}\right)^{*}$} \\
\cline { 2 - 5 } & Classe 1 & Classe 2 & Classe 3 & Comercial \\
\hline Gotejamento-Superfície do solo & $3,93 \mathrm{~b}$ & $14,64 \mathrm{a}$ & $17,31 \mathrm{a}$ & $35,88 \mathrm{a}$ \\
\hline Gotejamento-Subterrâneo & $8,39 \mathrm{a}$ & $11,42 \mathrm{a}$ & $8,57 \mathrm{~b}$ & $28,38 \mathrm{~b}$ \\
\hline
\end{tabular}

*Médias seguidas da mesma letra, na coluna, não diferem entre pelo teste de Scott-Knott a 5\% de probabilidade.

As produtividades de cebola por classes e a produtividade comercial para os tratamentos com irrigação por gotejamento com quatro linhas laterais com disposição na superfície do solo ou subterrânea são apresentadas na Tabela 3. Observa-se, pela Tabela 3, que não houve diferenças significativas entre os tratamentos.

Tabela 3. Produtividade de classes 1,2 e 3 e produtividade comercial, irrigada por gotejamento com quatro linhas laterais por canteiro, dispostas na superfície do solo e enterradas a $0,20 \mathrm{~m}$.

\begin{tabular}{lcccc}
\hline \multirow{2}{*}{ Tratamento } & \multicolumn{4}{c}{ Produtividade $\left(\mathrm{t} \mathrm{ha}^{-1}\right)^{*}$} \\
\cline { 2 - 5 } & Classe 1 & Classe 2 & Classe 3 & Comercial \\
\hline Gotejamento-Superfície do solo & $5,71 \mathrm{a}$ & $14,64 \mathrm{a}$ & $12,32 \mathrm{a}$ & $32,61 \mathrm{a}$ \\
\hline Gotejamento-Subterrâneo & $3,04 \mathrm{a}$ & $13,51 \mathrm{a}$ & $14,82 \mathrm{a}$ & $31,42 \mathrm{a}$ \\
\hline
\end{tabular}

"Médias seguidas da mesma letra, na coluna, não diferem entre pelo teste de Scott-Knott a 5\% de probabilidade.

As produtividades de cebola por classe e a produtividade comercial para todos os tratamentos com irrigação por gotejamento com duas, três e quatro linhas laterais, com disposição na superfície do solo ou subterrânea, são apresentadas na Tabela 4. No tratamento com três linhas laterais dispostas na superfície do solo, ocorreram as maiores produtividades de bulbos classe 3, correspondente ao produto de melhor valor comercial, com diferenças estatísticas significativas. A produtividade de bulbos classe 1, de menor valor comercial, foi menor nos tratamentos com três laterais dispostas na superfície do solo e com quatro linhas laterais subterrâneas. Para a produtividade de cebola classificada como classe 2, não houve diferenças significativas entre os tratamentos. Notou-se, ainda, que a menor produtividade comercial foi proporcionada pelo tratamento com duas linhas laterais subterrânea. 
Tabela 4. Produtividade de cebola classes 1, 2 e 3 e produtividade comercial, para todos os tratamentos.

\begin{tabular}{lcccc}
\hline \multirow{2}{*}{ Tratamento } & \multicolumn{3}{c}{ Produtividade (t ha $\left.{ }^{-1}\right)^{*}$} \\
\cline { 2 - 4 } & Classe 1 & Classe 2 & Classe 3 & Comercial \\
\hline Got. Superfície (2 Llat) & $5,89 \mathrm{~b}$ & $12,14 \mathrm{a}$ & $9,28 \mathrm{c}$ & $27,31 \mathrm{c}$ \\
\hline Got. Subterrânea (2 L lat) & $5,53 \mathrm{~b}$ & $12,67 \mathrm{a}$ & $1,57 \mathrm{~d}$ & $20,17 \mathrm{~d}$ \\
\hline Got. Superfície (3 L lat) & $3,93 \mathrm{c}$ & $14,64 \mathrm{a}$ & $17,31 \mathrm{a}$ & $35,88 \mathrm{a}$ \\
\hline Got. Subterrânea (3 L lat) & $8,39 \mathrm{a}$ & $11,42 \mathrm{a}$ & $8,57 \mathrm{c}$ & $28,38 \mathrm{c}$ \\
\hline Got. Superfície (4 L lat) & $5,71 \mathrm{~b}$ & $14,64 \mathrm{a}$ & $12,32 \mathrm{~b}$ & $32,61 \mathrm{~b}$ \\
\hline Got. Subterrânea (4 L lat) & $3,04 \mathrm{c}$ & $13,51 \mathrm{a}$ & $14,82 \mathrm{~b}$ & $31,42 \mathrm{~b}$ \\
\hline *Médias seguidas da mesma letra, na coluna, não diferem entre pelo teste de Scott-Knott a 5\% de probabilidade.
\end{tabular}

*Médias seguidas da mesma letra, na coluna, não diferem entre pelo teste de Scott-Knott a 5\% de probabilidade.

\section{CONCLUSÕES}

O sistema de irrigação por gotejamento com três linhas laterais por canteiro, dispostas na superfície do solo, garantiu maior produtividade de cebola classe 3 que possui melhor valor comercial.

O sistema de irrigação por gotejamento com linhas laterais subterrâneas por canteiro se mostrou contra indicado para a cultura da cebola.

\section{REFERÊNCIAS BIBLIOGRÁFICAS}

AKHTAR, M.E.; BASHIR, K.; KHAN, M.Z.; KHOKLAR, K.M. Effect of potash application on yield of different varieties of onion (Allium cepa L.). Asian Journal of Plant Science, Islamabad, v.1, n.4, p.324-325, 2002.

BANDEIRA, G. R. L.; QUEIROZ, S. O. P. de; ARAGÃO, C. A.; COSTA, N. D.; SANTOS, C. A. F. Desempenho agronômico de cultivares de cebola sob diferentes manejos de irrigação no submédio são Francisco. Irriga, Botucatu, v. 18, n. 1, p. 73- 84, 2013.

CHRISTOFIDIS, D. Como obter a sustentabilidade dos recursos hídricos na agricultura irrigada? Irrigação \& Tecnologia Moderna, Brasília, v.64, p.30-31. 2004.

COSTA, N. D.; PINTO, J. M.; YURI, J. E.; CALGARO, M.; CORREIA, R. C. Manejo de água e nutrientes na cultura da cebola sob irrigação por gotejamento. In: CONGRESSO NACIONAL DE IRRIGAÇÃO E DRENAGEM, 25., 2015, São Cristóvão Agricultura irrigada no Semiárido brasileiro: anais. São Cristóvão: ABID: Universidade Federal de Sergipe, 2015. 
COSTA, N. D., PINTO, J. M. A Cultura da Cebola. Brasília: Embrapa, 2012 p.117.

DUARTE, R. L. R.; VElOSO, M. E. C.; MELO, F. B.; ATHAYDE SOBRINHO, C.; RIBEIRO, V. Q.; SILVA, P. H. S. Produtividade de cultivares de cebola no Semiárido piauiense. Horticultura Brasileira, Brasília, v. 21, n. 1, p. 34-36, 2003. FILGUEIRA, F.A.R. Novo manual de olericultura: agrotecnologia moderna produção e comercialização de hortaliças. 3.ed. Viçosa, MG: UFV, 2008. 421p.

GONÇALVES, P. A. S.; WORDELL FILHO, J. A.; KURTZ, C. Efeitos da adubação sobre a incidência de tripes e míldio e na produtividade da cultura da cebola. Revista Agropecuária Catarinense, v.22, p.57-60, 2009.

INSTITUTO BRASILEIRO DE GEOGRAFIA E ESTATÍSTICA - IBGE/LSPA. 2015. Levantamento Sistemático da Produção Agrícola. Rio de Janeiro, v. 29, n. 3, p. 1-81.

MANTOVANI, E.C.; BERNARDO, S.; PALARETTI, L.F. Irrigação: princípios e práticas. Viçosa: UFV, 2006. 318p.

MAROUELLI, W. A. VIDIGAL, S. M.; COSTA, E. L da Irrigação e fertirrigação na cultura da cebola. In: SOUSA, V. F de; MAROUELLI, W. A.; COELHO, E. F.; PINTO, J. M.; In: Irrigação e fertirrigação em fruteiras e hortaliças.1 ed. Brasília: Embrapa Informação Tecnológica, 2011, p. 585-608.

MAROUELLI, W.A.; CALBO, A.G.; CARRIJO, O.A. Avaliação de sensores do tipo Irrigas ${ }^{\circledR}$ para o controle da irrigação em hortaliças cultivadas em substratos. Irriga, v.10, n.1, p.88-95, 2005.

MOHANTY, B. B.; DAS, J. N. Response of rabi onio cv. Nasik Red to nitrogen and potassium fertilization. Vegetable Science, Varanasi, v. 28, n1, p. 40-42, 2001.

PAPADOLOULOS, I. Regional Middle East and Europe project on nitrogen fixation and water balance studies. IAEA, Viena, 1993. 65p.

PASCHOLD, P.J.; MOHAMMED, A.; MAYER, N.Irrigas - a new simple soil moisture sensor for irrigation scheduling. Acta Horticulturae, v.664, p.521-527, 2004.

SHARMA, R.P. Effect of planting material, nitrogen and potash on bulb yield of rainy-season onion (Allium cepa). Indian Journal of Agronomy, New Delhi, v.37, n.4, p.868-869, 1992. 
SHOCK, C. C; SHOCK, C. B. Research, Extension, and Good Farming Practices Improve Water Quality and Productivity. Journal of Integrative Agriculture, 2012, v. 11, n. 1, p. 1430.

RAJPUT, T. B. S.; PATEL, N. Effect of subsurface drip irrigation on onion yield. Irrigation Science, v.27, p.97-108, 2009.

SOUZA, W, de J.; BOTREL, T. A.; COELHO, R. D.; NOVA. N. A. V. Irrigação localizada subsuperfical: gotejador convencional e novo protótipo. Revista Brasileira de Engenharia Agrícola e Ambiental. Campina Grande. v.16, n.8, p.811-819, 2012. 